\title{
Much Ado about Dosing: The Needs and Challenges of Defining a Standardized Cannabis Unit
}

\author{
Sebastian Jugla, ${ }^{a}$ Ruba Sajdeya ${ }^{a, c}$ Earl J. Morris ${ }^{b}$ Amie J. Goodin ${ }^{a, b}$ \\ Joshua D. Brown ${ }^{a, b}$ \\ aConsortium for Medical Marijuana Clinical Outcomes Research, University of Florida, Gainesville, FL, USA; \\ bDepartment of Pharmaceutical Outcomes \& Policy, Center for Drug Evaluation and Safety (CoDES), University of \\ Florida, Gainesville, FL, USA; 'Department of Epidemiology, University of Florida, Gainesville, FL, USA
}

\section{Key Points}

- A definition of a standardized cannabis unit is needed to accelerate research in medical cannabis and enable safe and effective use of medical cannabis products.

- A standardized cannabis unit should incorporate several factors, such as plant-related aspects (e.g., varied cannabinoid concentrations in Cannabis sativa) and product attributes, such as different administration routes and cannibinoid concentrations. Furthermore, different intentions for the use and desired subjective effects also influence the dose needed for the intended effect.

- Many barriers remain in defining a standardized unit for cannabis (e.g., different delivered doses and pharmacokinetics depending on the administration route) and pathophysiological factors that can impact the response to the therapy or side-effect profile.

- A recent proposal for a standardized tetrahydrocannabinol (THC) unit by Freeman and Lorenzetti, which defines a "Standard THC Unit" of $5 \mathrm{mg}$ THC, presents a sophisticated approach to support safe, nonmedical cannabis consumption within the same administration route. However, this approach may be limited when considering medical cannabis products, given a need to track efficacy and safety, a variety of products available, and the need to understand the composition of other cannabinoids.

- Further efforts in developing a standardized cannabis unit are needed to capture the medical cannabis perspective, possibly including the antagonizing effect of cannabidiol on THC, the role of the entourage effect, and the relationship between pharmacokinetic profiles and therapeutic effects of cannabis constituents.

\section{Keywords}

Cannabidiol · Tetrahydrocannabinol · Cannabis · Entourage effect $\cdot$ Standardized unit $\cdot$ Dosing $\cdot$ Pharmacokinetic

As research strives to catch up with the increasing prevalence of cannabis use, unit standardization of cannabis emerges as one of the most significant knowledge gaps and biggest challenges [1-4]. Several proposed approaches to standardize cannabis units resemble those implemented for alcohol. However, additional complexities related to cannabis hinder these efforts [2]. Previous strategies focused on measuring the quantity of consumed cannabis within the context of specific administration methods, such as a "standard joint equivalent" [5]. More recent approaches propose standardizing by the
(C) 2021 The Author(s)

Published by S. Karger AG, Basel

This is an Open Access article licensed under the Creative Commons Attribution-NonCommercial-4.0 International License (CC BY-NC) (http://www.karger.com/Services/OpenAccessLicense), applicable to the online version of the article only. Usage and distribution for commercial purposes requires written permission.
Correspondence to:

Joshua D. Brown, joshua.brown@ufl.edu 
Table 1. Needs and barriers for the implementation of a standardized cannabis unit

\begin{tabular}{lll}
\hline Factors & Need for implementing a standardized cannabis unit & Barriers to implementing a standardized cannabis unit \\
\hline $\begin{array}{lll}\text { Cannabis plant and } \\
\text { product factors }\end{array}$ & $\begin{array}{l}\text { - Varied cannabinoid concentrations in Cannabis sativa } \\
\text { and cultivation of high potency strains } \\
\text { - Available products with different routes of } \\
\text { administration }\end{array}$ & $\begin{array}{l}\text { - Different delivered doses and pharmacokinetics depending on the } \\
\text { administration routes } \\
\text { - Variability in other cannabinoids and terpenes concentrations can } \\
\text { impact the effect of cannabis (entourage effect) }\end{array}$ \\
\hline $\begin{array}{lll}\text { Clinical and user } \\
\text { factors }\end{array}$ & $\begin{array}{l}\text { - Intention of use (medicinal vs. recreational vs. both), } \\
\text { and desired subjective effect, influence the dose needed }\end{array}$ & $\begin{array}{l}\text { - Intention of use (recreational, medical, etc.) and desired subjective } \\
\text { for the intended effect lead to different relevant doses }\end{array}$ \\
& - Inability to titrate, in particular with edible forms & $\begin{array}{l}\text { - Pathophysiological factors or comedications impacting response to } \\
\text { therapy or side-effect profile (e.g., liver dysfunction, drug interactions, }\end{array}$ \\
& & $\begin{array}{l}\text { etc.) } \\
\text { - Different administration patterns can lead to pharmacokinetic } \\
\text { differences (e.g., number of puffs, length of inhalation, etc.) and } \\
\text { differences in overall exposure }\end{array}$ \\
\hline
\end{tabular}

amount of active pharmacological constituents, mainly tetrahydrocannabinol (THC) and cannabidiol (CBD) [6]. This commentary elaborates on the necessity for defining a standardized unit and evaluates the strengths and limitations of a recent proposal from Freeman and Lorenzetti [6], specifically related to medical cannabis and cannabinoids.

Freeman and Lorenzetti propose a "Standard THC Unit" of $5 \mathrm{mg}$ based on the premise that THC is the principal constituent responsible for the psychoactive effects of cannabis. The authors posit THC is the optimal standard unit to measure, especially with regards to safety. They suggest using this standard unit in product labeling and inclusion in the Lower Risk Cannabis Use Guidelines [7]. They argue that a standard THC unit of $5 \mathrm{mg}$ is a sufficiently low dose to minimize adverse events and unintentional excessive dosing while still offering noticeable psychotropic effects and meaningful quantity for users.

The potential for widespread adoption of a universal, standardized cannabis unit has broad implications for several key stakeholders, including researchers, clinicians, and patients. Current research is often limited by the lack of specific information on the cannabis products studied. A recent review of medical cannabis clinical research in the USA showed significant variability in the dose, route of administration, and THC/CBD content of cannabis products studied [8]. Clinical trials consistently report dose and administration route (typically peroral, oral, and sublingual routes). However, it is difficult to compare findings between different products used across studies and the products available to the broader public, especially via different administration routes. Furthermore, many observational studies lack specific information about cannabis dose, route, and product. As such, reliable evidence on the safety and effectiveness of cannabis products remains limited in the absence of unit standardization [9].

For clinicians and patients, a standardized cannabis unit guides initiating, maintaining, and adjusting cannabis therapy. Conversely, it may improve the identification and management of problematic cannabis use or cannabis use disorders, especially when multiple administration routes are used [10]. Widespread implementation of a standardized unit can improve patient education and clarify informational materials, which could ultimately mitigate overconsumption and allow for easier comparison among available products (Table 1) [11].

Several obstacles to defining a standardized cannabis unit persist due to the complex clinical and pharmacological nature of cannabis. One obstacle lies in the nature of the plant and its products. Cannabis sativa consists of more than 100 different cannabinoids, of which THC and CBD are the most researched. THC is assumed to be mainly responsible for the psychoactive effects of cannabis, whereas CBD has no known psychoactive effects but may have significant anti-inflammatory properties [12]. THC and CBD concentrations also differ widely between different cannabis varieties and preparations (e.g., cannabis flower, cannabis extract, or edibles). The pharmacokinetics of THC and CBD vary significantly by administration route, which impacts the total exposure to the cannabinoids and the timing and extent of side effects $[13,14]$. User factors such as the intention for the use (e.g., nonmedical or medical, underlying medical condition, etc.), subjective desired effects, varying administration patterns, pharmacogenomics, and drug-drug interactions further increase the difficulty in defining an appropriate standardized cannabis unit. 
While there is a clear need for a standardized cannabis unit and a consistent " $5 \mathrm{mg}$ of THC" unit is easily interpretable, there remain limitations to this approach. Freeman and Lorenzetti [6] standardized cannabis unit definition explicitly focuses on the safety of cannabis consumption and is analogous to using standardized alcohol consumption units. However, in contrast to the alcohol example, this approach is limited given cannabis is also used for medical purposes and has varied modes of use. This fact makes defining an effective dose just as important as determining a safe amount and tracking these doses across administration routes. CBD is also a key component in medical cannabis and cannabinoid products used alone or in combination with THC for various medical conditions, such as epilepsy and chronic pain. In fact, a recently published, consensus-based task force recommendation for treating chronic pain with medical cannabis based their treatment approaches on CBD doses, not THC $[15,16]$. Studies also suggest that CBD modulates the side-effect profile of THC. Thus, CBD amount and the THC:CBD ratio may be an equally important consideration for medical cannabis products. There is further evidence for the "entourage effect," which suggests that the concentration of other cannabinoids and noncannabinoids, such as terpenes, and phytochemicals are essential for therapeutic effects $[17,18]$. So, while a THCoriented definition promotes safety, it ignores the importance of $\mathrm{CBD}$, other compounds in cannabis, and the increasingly common use of cannabis for medical purposes.

Differences in administration practices and the resulting pharmacokinetics impose additional limitations to interpreting a standardized cannabis unit. For example, the oral bioavailability of THC can be as low as $6 \%$ over a 6-8 h period, whereas the bioavailability of inhaled THC ranges from 10 to $35 \%$ over a $15-60$ min period $[19,20]$. Thus, due to pharmacokinetic parameters like absorption and metabolism, the same amount of THC and CBD is not comparable across different administration routes. For example, an edible with "2 THC units" versus a vaporized product with the same number of THC units are unlikely to lead to similar effects because a different dose is delivered to the body and over a different exposure period. Such standardization does promote dose titration within the same administration route for users and may encourage more clear instructions to patients. Communication to patients about the differences between the concentration and the actual "delivered dose" and timing of that dose is critical to avoid accidental intoxication or underdosing when switching or using multiple products.
Consensus regarding a standardized cannabis unit is of utmost importance to accelerate research in medical cannabis and enable safe and effective use of cannabis products. Future proposals must consider the antagonizing effect of CBD on THC, the role of the entourage effect, and the relationship between pharmacokinetic profiles and therapeutic effects of cannabis constituents. The proposed standardized cannabis unit from Freeman and Lorenzetti presents an approach to support safe cannabis consumption, at least within the same administration route and especially for nonmedical use where safety is the focus. However, it is unlikely that a "one size fits all" definition will capture both nonmedical and medical use of cannabis and may be insufficient for constructing comparisons between administration routes. Updated proposals for a standardized cannabis unit should therefore consider the increasing use of medical cannabis and cannabinoids, the role of $\mathrm{CBD}$, and the administration route to incorporate data for both safety and therapeutic effects.

\section{Acknowledgement}

The authors are supported by State of Florida appropriations to the Consortium for Medical Marijuana Clinical Outcomes Research (mmjoutcomes.org). Evidence in Context is part of the outreach effort of the Consortium for Medical Marijuana Clinical Outcomes Research to examine and discuss implications of research into cannabis and cannabinoids for clinical practice, thus providing a translational approach to these studies to make clear, concise, and actionable evidence available for clinicians and patients.

\section{Conflict of Interest Statement}

The authors have no conflicts of interest to declare.

\section{Funding Sources}

The authors did not receive any funding.

\section{Author Contributions}

S.J., R.S., and J.B. conceptualized and drafted the work. E.M. and A.G. drafted and revised. All approved the final version. 


\section{References}

1 National Institute on Drug Abuse. What is the scope of marijuana use in the United States? Available from: https://www.drugabuse.gov/ publications/research-reports/marijuana/ what-scope-marijuana-use-in-united-states. Accessed 2020 May 19.

2 National Academies of Sciences, Engineering, and Medicine. Challenges and barriers in conducting cannabis research. National Academies Press (US); 2017. Available from: https: //www.ncbi.nlm.nih.gov/books/ NBK425757/. Accessed 2020 Feb 29.

3 National Academies of Sciences, Engineering, and Medicine. Recommendations to support and improve the cannabis research agenda. National Academies Press (US); 2017. Available from: https://www.ncbi.nlm.nih.gov/ books/NBK425745/. Accessed 2021 Mar 30.

4 National Institute on Drug Abuse. Request for information: standard unit dose of THC. National Institute on Drug Abuse [published 2020 Mar 23]. Available from: https://www. drugabuse.gov/about-nida/norasblog/2020/03/request-information-standardunit-dose-thc. Accessed 2021 Mar 30.

5 Callaghan RC, Sanches M, Benny C, Stockwell T, Sherk A, Kish SJ. Who consumes most of the cannabis in Canada? Profiles of cannabis consumption by quantity. Drug Alcohol Depend. 2019;205:107587.

6 Freeman TP, Lorenzetti V. "Standard THC units": a proposal to standardize dose across all cannabis products and methods of administration. Addiction. 2020;115:1207-16.
7 Fischer B, Russell C, Sabioni P, van den Brink W, Le Foll B, Hall W, et al. Lower-risk cannabis use guidelines: a comprehensive update of evidence and recommendations. Am J Public Health. 2017;107:e1-12.

8 Jugl S, Okpeku A, Costales B, Morris EJ, Alipour-Haris G, Hincapie-Castillo JM, et al. A mapping literature review of medical cannabis clinical outcomes and quality of evidence in approved conditions in the USA from 2016 to 2019. Medical Cannabis and Cannabinoids. 2021.

9 Adams TN, Butt YM, Batra K, Glazer CS. Cobalt related interstitial lung disease. Respir Med. 2017;129:91-7.

10 Loflin MJE, Kiluk BD, Huestis MA, Aklin WM, Budney AJ, Carroll KM, et al. The state of clinical outcome assessments for cannabis use disorder clinical trials: a review and research agenda. Drug Alcohol Depend. 2020; 212:107993

11 Goodman S, Hammond D. Does unit-dose packaging influence understanding of serving size information for cannabis edibles? J Stud Alcohol Drugs. 2020;81:173-9.

12 National Academies of Sciences, Engineering, and Medicine. The health effects of cannabis and cannabinoids: the current state of evidence and recommendations for research. Washington, DC: The National Academies Press; 2017. p. 486.
13 Brown JD, Winterstein AG. Potential adverse drug events and drug-drug interactions with medical and consumer cannabidiol (CBD) use. J Clin Med. 2019;8:989.

14 Brown JD. Potential adverse drug events with tetrahydrocannabinol (THC) due to drugdrug interactions. J Clin Med. 2020;9.

15 Lattanzi S, Brigo F, Trinka E, Zaccara G, Cagnetti C, Del Giovane C, et al. Efficacy and safety of cannabidiol in epilepsy: a systematic review and meta-analysis. Drugs. 2018;78: 1791-804.

16 PAINWeek abstract book 2020, abstract 19 Postgrad Med 2020;132:17-8.

17 Ben-Shabat S, Fride E, Sheskin T, Tamiri T, Rhee MH, Vogel Z, et al. An entourage effect: inactive endogenous fatty acid glycerol esters enhance 2-arachidonoyl-glycerol cannabinoid activity. Eur J Pharmacol. 1998;353:2331.

18 Hanuš LO, Hod Y. Terpenes/terpenoids in cannabis: are they important? Medical Cannabis and Cannabinoids. 2020;3:25-60.

19 Lucas CJ, Galettis P, Schneider J. The pharmacokinetics and the pharmacodynamics of cannabinoids. Br J Clin Pharmacol. 2018;84(11): 2477-82.

20 Naef M, Russmann S, Petersen-Felix S, Brenneisen R. Development and pharmacokinetic characterization of pulmonal and intravenous delta-9-tetrahydrocannabinol (THC) in humans. J Pharm Sci. 2004;93:1176-84. 